\title{
Successful Treatment of Advanced Ovarian Cancer with Thermochemotherapy and Adjuvant Immune Therapy
}

\author{
R. Kleef S. Kekic N. Ludwig \\ Dr. Kleef - Hyperthermie, Stiftung Integrative Onkologie, Vienna, Austria
}

\section{Key Words}

Ovarian cancer - Thermo-chemotherapy - Adjuvant immune therapy ·

Whole-body hyperthermia $\cdot$ Dendritic cell therapy

\begin{abstract}
We report on a 4-year progression-free survival of a 54-year-old female first diagnosed in December 2007 with advanced bilateral ovarian cancer FIGO IIIc, disseminated peritoneal carcinosis and malignant diaphragm invasion. Treatment started in January 2008 with 6 cycles of Taxol $175 \mathrm{mg} / \mathrm{m}^{2} /$ carboplatin AUC 5 in 3-week intervals. Twenty-four hours following each chemotherapy session, fever-range long-duration whole-body hyperthermia (WBH) was performed at the temperature plateau of $40^{\circ} \mathrm{C}$ body core temperature for $6 \mathrm{~h}$. Three months after completion of chemotherapy, 4 more long-duration WBH procedures were performed in monthly intervals. Importantly, long-duration WBH was paralleled with intradermal vaccination of autologous dendritic cells. No other treatment was given to the patient. Four years following the first diagnosis, the patient is still in complete remission with no evidence of disease.
\end{abstract}

\section{Background}

Ovarian cancer usually has a poor prognosis, even more so the advanced-stage ovarian cancer described in this case report, despite radical surgery and adjuvant chemotherapy. For women with advanced ovarian cancer, the prognosis largely depends on the extent of tumor mass reduction at the initial surgery. Complete resection confers significantly longer survival (median 5 years) than incomplete resection. Because of the benefit of cytoreductive surgery and the efficacy of platinum plus paclitaxel first-line chemotherapy, these procedures have been established as the standard treatments for advanced epithelial ovarian cancer. In spite of this, long-term 
survival has not improved. In the present case, disseminated peritoneal carcinosis and metastatic spread to the kidney and diaphragm represented far-advanced disease with an expected overall 5-year median survival of $<23 \%$ [1].

\section{Case Presentation}

Staging at initial diagnosis in December 2007 was G3, pT3c, N0 (0/4), Mx, R2. N. ovarii bilateralis with disseminated peritoneal carcinosis, cystic douglas cavity tumor with adhesion to the rectum and sigma, renal capsule metastasis, diaphragm metastasis above the liver in form of metastasis plate and metastasis in the omentum majus. Postoperative histology revealed low-differentiated diffuse and nodular partially papillary and partially solid-serous papillary adenocarcinoma of both ovaries G3.

Surgical resection with median lower and upper laparotomy was performed in December 2007, including bilateral extirpation of the uterus and adnexa with lymphadenectomy, omentum majus resection, excision of the mesorectum with descend-rectostomy in a double-stapling procedure, disseminated metastases in the peritoneum and metastatic resection of lesions adherent to the colon ascendens, the capsule of Glisson and the diaphragm.

Clinically, the patient presented with a painful cystic tumor in the rectovaginal area and moderate inflammatory and high tumor markers with a Karnofsky score of $80 \%$.

Treatment

Initially, the patient underwent debulking surgery in December 2007 as described above. Signed informed consent was obtained from the patient. Adjuvant locoregional hyperthermia with a radiofrequency of $13.56 \mathrm{MHz}$ (Oncotherm ${ }^{\mathrm{EHY}}{ }^{2000}$ ) was performed over 2 weeks three times a week before the initiation of chemotherapy.

From January to May 2008, adjuvant chemotherapy treatment was started with 6 cycles of Taxol $175 \mathrm{mg} / \mathrm{m}^{2} /$ carboplatin AUC 5 in 3-week intervals. Twenty-four hours following each chemotherapy session, fever-range long-duration whole-body hyperthermia (WBH; Heckel HT2000, Heckel Medizintechnik, Esslingen, Germany) was performed at the temperature plateau of $40^{\circ} \mathrm{C}$ body core temperature for $6 \mathrm{~h}$ under intensive care monitoring standards and light neuroleptic analgesia. The rationale and methodology of long-duration WBH have been described elsewhere [2-4].

From July to October 2008, 3 months after completion of chemotherapy, 4 more long-duration WBH procedures were performed in monthly intervals. Importantly, again long-duration WBH was paralleled with intradermal vaccination of autologous primed dendritic cells (DC; LANEX-DC, Cabion Technologies, Ulm, Germany) [5, 6]. In brief, LANEX-DC (long antigen exposition DC) were produced according to the GMP guidelines as follows:

Peripheral blood mononuclear cells (PBMC) were isolated from $250 \mathrm{ml}$ of heparinized venous blood of the patient by density-gradient centrifugation. PBMC were seeded in 6 -well plates and after $2 \mathrm{~h}$ the non-adherent cells were removed. Adherent cells were cultured in RPMI 1640 supplemented with $10 \%$ of the patient serum and L-glutamine in the presence of rh-GM-CSF and rh-IL- 4 for 7 days. Maturation of monocyte-derived DC was induced by adding rh-IL-1 $\beta$, rh-TNF- $\alpha$ and rh-IL- 6 . After $24 \mathrm{~h}$, monocyte-derived DC were harvested. The cells were suspended in $1 \mathrm{ml}$ of sterile saline solution containing $10 \%$ autologous serum and administered by intracutaneous injection in the abdominal cutis near the inguinal lymph nodes (500 $\mu \mathrm{l}$ of cell suspension on each side). Vaccination was administered subcutaneously at two locations near the groin lymph nodes.

Outcome and Follow-Up

Restaging by full laboratory analysis including TM CA125, CEA and CT of the abdomen was performed in 6-month intervals. The patient is now, 4 years following the initial diagnosis, free of any signs of cancer in lasting complete remission. The CT of the abdomen in May and October 2008 and June and December 2009 all showed no signs of cancer recurrence. The TM CA125, which could be taken as laboratory surrogate marker, was still in the normal range in November 2011. An analysis of circulating tumor cells from the blood (PCR detection of tumor-specific gene expression) in December 2009 was negative ( $\underline{\underline{\text { fig. }} \mathbf{1}}$ ). 


\section{Discussion}

To our knowledge, this is the first advanced epithelial ovarian cancer case treated with a combination of thermochemotherapy and DC and followed up for 4 years without any evidence of disease recurrence.

There is a rising interest in the use of hyperthermia for the treatment of cancer [7]. Basically, locoregional and WBH techniques have to be differentiated. The first is becoming increasingly established in teaching university settings and is also widely used by many practices and clinics, mainly in Germany. The latter also has a longstanding tradition and has been introduced mainly by Heckel and von Ardenne in Germany, and Robins in the USA. In the 80s and 90s of the last century, some studies investigated the effects, treatment efficacy and immunological findings of hightemperature $\left(41.8-42^{\circ} \mathrm{C}\right) \mathrm{WBH}$ in different cancers. Most studies were conducted in small patient numbers and in combination with chemotherapy. The clinical response rates in most of these studies of high temperature yielded marginal clinical benefit regarding (prolonged) progression-free survival or overall survival time. On the other hand, more moderate, fever-like temperatures clearly have been associated with lowered cancer incidence and remission of cancer [8].

Therefore, the use of fever-range $\left(39.5-40.0^{\circ} \mathrm{C}\right)$ prolonged $\mathrm{WBH}(4-6 \mathrm{~h}$ at temperature plateau) has been mainly researched since the 90 s of the last century by Bull and co-workers at the Division of Oncology, University of Texas Medical School [9] and Repasky and co-workers at the Roswell Park Cancer Centre [10]. An impressing amount of preclinical and to a smaller amount also clinical publications strongly confirmed the induction of immunological mechanisms relevant in the complex network of anti-tumor processes induced by WBH; moreover, the use of WBH in combination with different chemotherapy regimens demonstrated clear advantages over the use of chemotherapy alone. Importantly, timing and dosing of the applied methods and substances played a crucial role, pointing to the necessity to develop metronomic therapies in the treatment of patients suffering from cancer.

There is a smaller in vitro and a larger in vivo part investigating different dose and time schedules of the combination of fever-range long-duration WBH with chemotherapy. The basic mechanism of the chemotherapy anti-cancer effects is the modification of cellular DNA by forming chemotherapy DNA adducts, altering the structure and function of proteins that interact directly with the genome [11]. Earlier studies demonstrated that chemotherapy acts synergistically with heat in vitro and in vivo.

Hyperthermic intraperitoneal chemotherapy is under continuous evaluation as a potential treatment for ovarian cancer. Our approach with thermochemotherapy may be a concurrent methodology.

\section{Implications}

The results of this case report clearly underline and call for the necessity of further research and the rapid transfer of these findings into well-designed clinical phase II/III trials. 


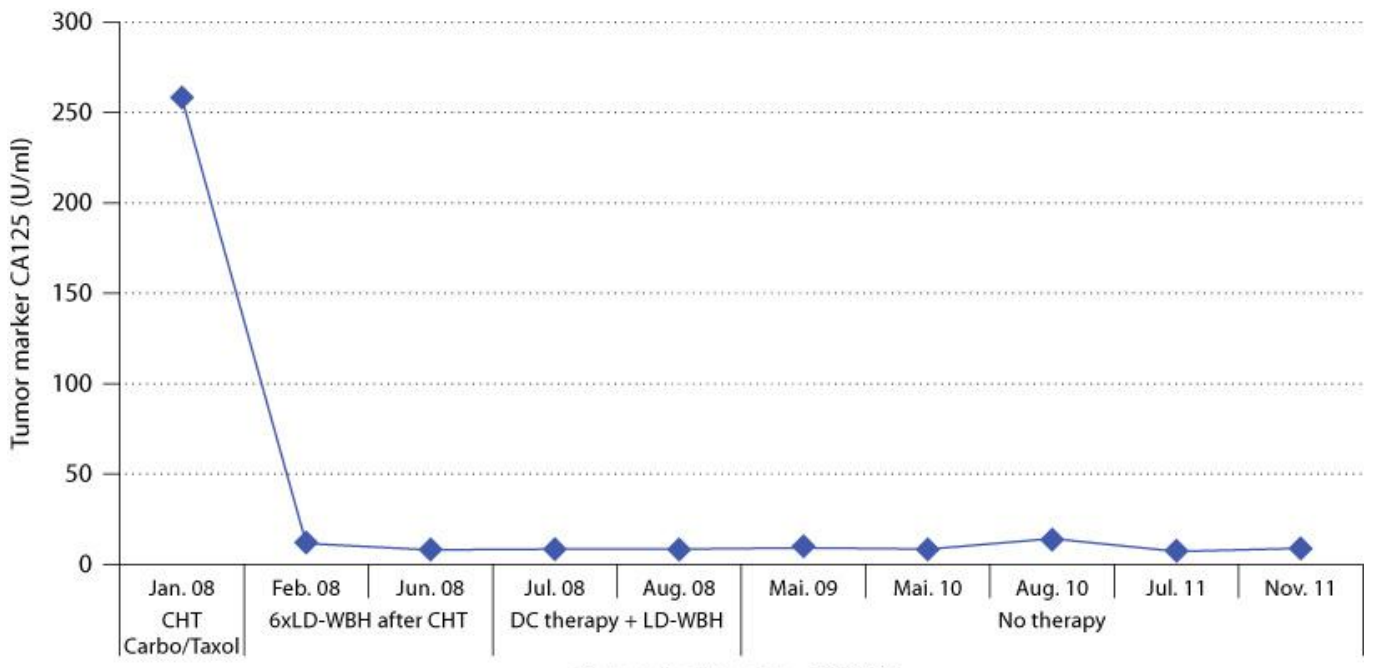

Determination date of CA125

Fig. 1. Development of tumor marker CA125. CHT = Chemotherapy; LD-WBH = long-duration WBH.

\section{References}

1 Ovary and primary peritoneal carcinoma; in Edge SB, Byrd DR, Compton CC, Fritz AG, Greene FL, Trotti A (eds): AJCC Cancer Staging Manual. 7th ed. New York, Springer, 2010, pp 493-506.

$\longrightarrow 2$ Skitzki JJ, Repasky EA, Evans SS: Hyperthermia as an immunotherapy strategy for cancer. Curr Opin Investig Drugs 2009;10:550-558.

-3 Peer AJ, Grimm MJ, Zynda ER, Repasky EA: Diverse immune mechanisms may contribute to the survival benefit seen in cancer patients receiving hyperthermia. Immunol Res 2010;46:137-154.

-4 Kleef R: Journal Club Commentary. Forsch Komplementmed 2011;18:97-101.

5 Banchereau J, Steinmann RM: Dendritic cells and the control of immunity. Nature 1998;392:245-252.

-6 Goyne H, Stone PJ, Cannon MJ: Combinatorial strategies for alleviation of tumor-associated immune suppression and therapeutic vaccination against ovarian cancer. Immunotherapy 2011;3:805-807.

7 Viglianti BL, Stauffer P, Repasky EA, Jones E, Vujaskovic Z, Dewhirst M: Hyperthermia; in Hong WK, Kufe DW, Bast RC, Hait W, Pollock RE, Weichselbaum RR, Holland JF, Frei E (eds): Holland-Frei Cancer Medicine, ed 8. BC Decker, Hamilton, 2011, pp 528-540.

8 Kleef R, Jonas WB, Knogler W, Stenzinger W: Fever, cancer incidence and spontaneous remissions. Neuroimmunomodulation 2001;9:55-64.

-9 Rowe RW, Strebel FR, Proett JM, Deng W, Chan D, He G, Siddik Z, Bull JM: Fever range whole body thermal therapy with oxaliplatin: a curative regimen in a pre-clinical breast cancer model. Int J Hyperthermia 2010;26:565-576.

10 Peer AJ, Grimm MJ, Zynda ER, Repasky EA: Diverse immune mechanisms may contribute to the survival benefit seen in cancer patients receiving hyperthermia. Immunol Res 2010;46:137-154.

11 Chaney SG, Campbell SL, Bassett E, Wu Y: Recognition and processing of cisplatin- and oxaliplatin-DNA adducts. Crit Rev Oncol Hematol 2005;53:3-11. 